\title{
Cone-beam CT versus Multidetector CT in Postoperative Cochlear Implant Imaging: Evaluation of Image Quality and Radiation Dose
}

\author{
(D) R.A. Helal, (D) R. Jacob, (D) M.A. Elshinnawy, (D) A.I. Othman, (DI.M. Al-Dhamari, (DD.W. Paulus, and (D)T.T. Abdelaziz
}

\begin{abstract}
BACKGROUND AND PURPOSE: Cone-beam CT is being increasingly used in head and neck imaging. We compared cone-beam CT with multidetector $C T$ to assess postoperative implant placement and delineate finer anatomic structures, image quality, and radiation dose used.
\end{abstract}

MATERIALS AND METHODS: This retrospective multicenter study included 51 patients with cochlear implants and postoperative imaging via temporal bone cone-beam CT ( $n=32$ ears) or multidetector CT ( $n=19$ ears) between 2012 and 2017. We evaluated the visualization quality of single electrode contacts, the scalar position of the electrodes, cochlear walls, mastoid facial canal, metallic artifacts (using a 4level visual score), and the ability to measure the insertion angle of the electrodes. The signal-to-noise ratio and radiation dose were also evaluated.

RESULTS: Cone-beam CT was more sensitive for visualizing the scalar position of the electrodes $(P=.046)$, cochlear outer wall $(P=.001)$, single electrode contacts $(P<.001)$, and osseous spiral lamina $(P=.004)$ and had fewer metallic artifacts $(P<.001)$. However, there were no significant differences between both methods in visualization of the modiolus $(P=.37)$, cochlear inner wall $(P>$.99), and mastoid facial canal wall $(P=.07)$ and the ability to measure the insertion angle of the electrodes $(P>.99)$. The conebeam $C T$ group had significantly lower dose-length product $(P<.001)$, but multidetector $C T$ showed a higher signal-to-noise ratio in both bone and air $(P=.22$ and $P=.001)$.

CONCLUSIONS: Cone-beam CT in patients with cochlear implants provides images with higher spatial resolution and fewer metallic artifacts than multidetector $\mathrm{CT}$ at a relatively lower radiation dose.

ABBREVIATIONS: $\mathrm{CBCT}=$ cone-beam $\mathrm{CT} ; \mathrm{Cl}=$ cochlear implant; $\mathrm{MDCT}=$ multidetector $\mathrm{CT}$

A cochlear implant (CI) as a treatment option for profound sensorineural hearing loss has increased remarkably in recent years. This increase can be partly attributed to the innovative diagnostic radiologic procedures. These procedures facilitate preoperative and intraoperative processes and enhance postoperative outcomes. ${ }^{1}$ Preoperative assessment of the temporal bone and inner ear structures is crucial to check the feasibility of implantation and predict the outcome. Usually both MR imaging and multidetector CT (MDCT) are used for assessment. ${ }^{2}$ Intraoperative imaging is usually reserved for cases with severe anatomic abnormalities and during minimally invasive procedures to guide the

Received April 17, 2020; accepted after revision September 15.

From the Radiodiagnosis Department (R.A.H., M.A.E., A.I.O., T.T.A.), Ain Shams University, Cairo, Egypt; HNOplus (R.J.), Höhr-Grenzhausen, Germany; and Institute for computational visualistics (I.M.A.-D., D.W.P.), Koblenz University, Koblenz, Germany. Please address correspondence to Rania Helal, MD, Radiodiagnosis Department, Faculty of Medicine, Ain Shams University, 38 Ramsis street, Abbassia Square, PO Box 11381, Cairo, Egypt; e-mail: raniahelal@med.asu.edu.eg; @20f81fe9c71b44c

http://dx.doi.org/10.3174/ajnr.A6894 electrode placement and reduce the duration of the operation using fluoroscopy or mobile radiography. ${ }^{3}$

As in most cases, the use of MR imaging after a CI must be limited, so the electrode position is usually assessed using conventional $\mathrm{x}$-ray or $\mathrm{MDCT}^{3}$ An important postoperative assessment is to ensure the proper positioning of the electrode inside the cochlea and check its insertion depth (which are important factors for the first activation and follow-up) as well as to assess the scalar position (the proper location being in the scala tympani near the nerve endings), distance of the first contact from the round window (optimal, 3$4 \mathrm{~mm}$ ), and distance from the electrode to the modiolus or lateral wall. Any complications such as electrode kinking, looping, or dislocation; presence of extracochlear electrodes; inner ear trauma; or osseous spiral lamina injury should be also assessed. ${ }^{4-6}$

These assessments post-CI require optimal imaging techniques. Conventional x-ray can localize the implant position but does not provide much detail and is, therefore, inadequate. MDCT provides more anatomic details; however, it has remarkable metallic artifacts. ${ }^{3}$ 
Cone-beam CT (CBCT) is becoming increasingly popular for head and neck imaging despite its low contrast resolution, which precludes the examination of soft-tissue pathology, because of its high spatial resolution and relatively low radiation dose. In the field of CIs, the importance of CBCT is increasing due to its provision of more accurate details and fewer metallic artifacts. ${ }^{7-14}$

CBCT involves a rotating gantry to which an x-ray source (with a divergent cone-shaped radiation) and flat panel detector are fixed. During complete/partial rotation of the gantry, multiple sequential planar projection images of the FOV are obtained, which differ from the helical progression of the fan-shaped radiation used in MDCT devices. Thus, CBCT imaging requires a relatively longer duration compared with MDCT, which increases the overall risk of motion artifacts. ${ }^{15}$

The accuracy of radiologic implant assessment is also important for improving surgical skills (correct electrode positioning) and CI fitting procedures. Auditory outcome depends on the correct scalar position of the electrode in the scala tympani, without scalar translocation. Furthermore, these data help in the development of new devices, more accessible surgical techniques, and better follow-up of patients. ${ }^{16}$ Recently, the combination of postoperative $\mathrm{CBCT}$ with preoperative MR imaging has shown very good results in the assessment of electrode position. ${ }^{17}$

The purpose of this study was to compare CBCT with MDCT post-CI for radiologic evaluation of important finer anatomic details around the implant, electrode radiologic assessment, and assessment of metallic artifacts, signal-to-noise ratio, and radiation doses used.

\section{MATERIALS AND METHODS Study Design and Population}

This was a retrospective multicenter study approved by Ain Shams University institutional review board (approval number MD237/ 2017). We conducted a retrospective review from January 2012 to December 2017 using our institution's CI data base and a public human cochlea data base to retrieve the postoperative imaging data of patients who underwent CI surgery and postoperative MDCT (from our institution) or CBCT (from the public data base). Exclusion criteria were the presence of a morphologically abnormal cochlea, severe motion artifacts distorting the image quality, and inadequate data about the type of electrode inserted. For patients undergoing bilateral CI, only the right ear was included (for statistical reasons). Fifty-one patients were identified and included, regardless of their age or sex. Patients' demographic data and data about the types of electrodes inserted were collected.

\section{Radiologic Examination and Analysis}

CBCT Group. This group was scanned using the CBCT 3D Accuitomo 170 (J. Morita). Each ear was imaged separately using 90-kV tube voltage and 5-mA current, with a high-resolution mode (Hi-Res J.Morita) with a rotation of $180^{\circ}$. A voxel size of $0.125 \mathrm{~mm}$ and an ROI of $80 \times 80 \times 80 \mathrm{~mm}$ were used. Images were reconstructed with filtered back-projection using the G_001 reconstruction algorithm.

MDCT Group. The MDCT group was scanned using the MDCT Somatom Definition Flash (dual-source 64-detector row scanner; Siemens) with single-energy automated tube- voltage selection (CARE $\mathrm{kV}$; Siemens). The quality reference tube voltage was $120 \mathrm{kV}$, providing an acquisition of $100 \mathrm{kV}$. Automated tube modulation (CARE Dose4D; Siemens) was used, with a quality reference tube current of $375 \mathrm{mAs}$. Images were obtained using a beam collimation $=0.5 \mathrm{~mm}$, rotation time $=1$ second, FOV $=240 \mathrm{~mm}$, section thickness $=0.6 \mathrm{~mm}$, section interval $=0.3 \mathrm{~mm}$, and pitch $=0.8$. Reconstruction was performed using the Hr60 kernel.

The obtained images were anonymized and examined by 2 experienced neuroradiologists with 5 and 15 years of experience until consensus was reached. The images were processed in the coronal, sagittal, and cochlear views. The window width and level were 3500 and 500, which were adjusted according to the observers' preferences for optimal visualization of the cochlear structures near the metallic artifacts.

\section{Qualitative Image Analysis and Analysis of Electrode Positions} mage sharpness was defined by identification of important anatomic details, such as the cochlear inner and outer walls, osseous spiral lamina, modiolus, and the mastoid facial nerve canal wall. Each image was also evaluated for the precise scalar localization of the inserted electrode, its insertion angle, and visualization of single electrode contacts. The electrode insertion angle was measured using the method suggested by Pearl et $\mathrm{al}^{18}$ (Fig 1). Its measurement ability was categorized into a 2-level score $(0=$ not measurable; $1=$ measurable). The quality of visualization of the other details was reported according to a 4 -level score $(0=$ not visualized, $1=$ barely visualized, 2 = well-visualized, 3 = perfectly visualized). Scoring of metallic artifacts was also performed (ranging from $0=$ markedly affecting the diagnosis to $3=$ minimal metallic artifacts).

\section{Quantitative Image-Quality Analysis}

The signal-to-noise ratio was calculated for each group; for calculation, 2 ROIs measuring approximately $4 \mathrm{~mm}^{2}$ were used. One was at the bone surrounding the cochlea (posterior-medial to the basal turn), and the other was in the air in the external auditory canal. The signal was defined as the mean ROI value, while the SD was defined as image noise; the signal-to-noise ratio was calculated as the mean ROI signal value divided by the SD. ${ }^{19}$

Patient Dose Analysis. The dose-length product was analyzed; the calculation was according to the manufacturer-specific protocols, and the output was via the device. Separate measurements were not performed.

Statistical Analysis. Data were coded and processed using the Statistical Package for the Social Sciences (SPSS Statistics for Windows, Version 23.0; IBM). Quantitative data were presented mainly as medians with interquartile ranges, when not normally distributed. Qualitative variables were presented as numbers and percentages. The $\chi^{2}$ test was used for comparison of $\geq 2$ groups of categoric variables. The Monte Carlo test was used as correction for the $\chi^{2}$ test when $>20 \%$ of the cells had a count of $<5$ in the tables. For comparison of the dose measurements and signal-to-noise ratio (quantitative data with nonparametric distribution), the Mann-Whitney test was used. A $P$ value $\leq .05$ was considered statistically significant, with a confidence interval of $95 \%$. 


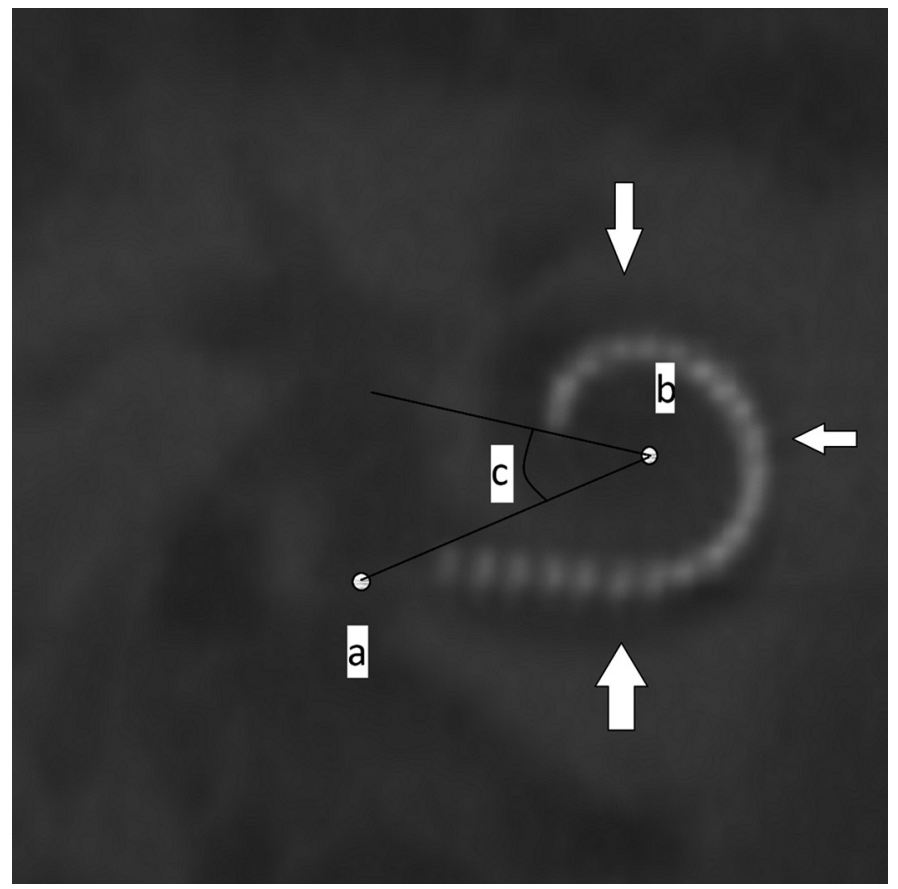

FIG 1. Coronal oblique (cochlear view with thick MPR $=5 \mathrm{~mm}$ ) $C B C T$ image showing the measurement of the angle of insertion between the deepest electrode and the reference line joining the insertion point (round window/cochleostomy center) (a) and the center of a circle formed by the 3 most apical electrodes (b). The insertion angle $=360^{\circ}-$ (c). The cochlear lateral wall is also well-visualized in this thick MPR coronal view (white arrows).

\section{Table 1: Different electrodes with intercontact distancing}

\begin{tabular}{lccc}
\multicolumn{1}{c}{ Electrodes } & $\begin{array}{c}\text { Intercontact } \\
\text { Distancing }(\mathbf{m m})\end{array}$ & $\begin{array}{c}\text { CBCT Group } \\
(\boldsymbol{n}=32)\end{array}$ & $\begin{array}{c}\text { MDCT Group } \\
(\boldsymbol{n}=19)\end{array}$ \\
\hline Widely spaced contacts & & & 0 \\
FLEX28 $^{\mathrm{a}}$ & 2.1 & 3 & 2 \\
FLEX-Synchrony-Medium $^{\mathrm{a}}$ & 1.9 & 2 & 1 \\
HiRes MIDSCALA $^{\mathrm{b}}$ & 0.975 & 3 & 0 \\
Cl422 $^{* \mathrm{c}}$ & $0.85-0.95$ & 1 & 0 \\
Narrowly spaced contacts & & & 0 \\
CI24RE-CA $^{\mathrm{c}}$ & Nonuniform 0.4-0.8 & 9 & 1 \\
CI512-CA $^{\mathrm{C}}$ & Nonuniform 0.4-0.8 & 3 & 15 \\
CI532-CA $^{\mathrm{c}}$ & 0.6 & 11 & \\
CI24RE-ST $^{\mathrm{C}}$ & 0.75 & 0 & \\
\hline
\end{tabular}

Note:-CA indicates contour advance; ST, straight electrode.

${ }^{a}$ MED-EL.

${ }^{\mathrm{b}}$ Advanced Bionics.

${ }^{c}$ Cochlear.

All distances were obtained from the portfolios of the electrodes ( ${ }^{*}$ except for Cl422 cited from Bennink et al. ${ }^{29}$ )

\section{Qualitative Image Analysis and Analysis of Electrode Positions}

The results of comparison between both groups regarding visualization of the finer anatomic details and electrode positioning are shown in Tables 2 and 3 (Fig 2). Scalar positioning of electrodes inside the cochlea was identified in both groups, except for 1 ear in the MDCT group (strong metallic artifacts). In the CBCT group $(n=32)$, electrodes in $63 \%$ of ears $(n=20)$ were inserted in the scala tympani (2 were barely visualized), $9 \%(n=3)$ were in the scala vestibuli (1 was barely visualized), and $28 \%(n=9)$ had scalar dislocation (1 was barely visualized), whereas in the MDCT group $(n=19), 79 \%(n=15)$ were inserted in the scala tympani (1 was barely visualized $), 11 \%(n=2)$ were in the scala vestibuli (1 was barely visualized), $5 \%(n=1)$ had scalar dislocation, and $5 \%$ were nonassessable $(n=1)$. The electrodes with widely spaced contacts showed perfectly visualized contacts $(n=3)$ in the MDCT group, despite the higher metallic artifacts and were perfectly to well-visualized $(n=7$ and $n=2$, respectively) in the CBCT group. The narrowly spaced contacts were barely visualized $(n=6)$ and were not visualized $(n=10)$ in the MDCT group compared with those in the CBCT group: wellvisualized $(n=9)$, barely visualized $(n=13)$, and not visualized $(n=1)$. The barely-to-nonvisualized electrodes in the CBCT group were mainly Cochlear CI532 and CI512.

\section{Quantitative Image-Quality Analysis}

The results of comparison of the signal-to-noise ratio between both

\section{RESULTS}

A total of 51 ears that received CIs were included in the final analysis. Thirty-two patients underwent postoperative СВСТ $($ females $=13[40.6 \%]$, males $=19[59.4 \%]$ with median age $=56$ years [interquartile range, 46-0.5 years] and range $=13-84$ years), and 19 patients underwent postoperative MDCT $($ females $=11[57.9 \%]$, males $=8[42.1 \%]$ with median age $=4$ years [interquartile range, 3-6 years] and range $=1-17$ years). The average exposure time for the CBCT protocol was 15.8 seconds, while that for MDCT was 4 seconds. Details of the implanted electrodes are shown in Table 1. groups are shown in Table 4.

\section{Dose Analysis}

There was a statistically significant 'difference between both groups in terms of the dose-length product values. The median (interquartile range) in the CBCT group was 93 (47.693) with a range of $47.6-93 \mathrm{mGy}^{*} \mathrm{~cm}$, while in the MDCT group, the median was (interquartile range) $=387.5(206-$ $527.75)$ with a range of $179-650 \mathrm{mGy}^{*} \mathrm{~cm}(P<.001)$. The difference between the maximum doses encountered in both groups was $557 \mathrm{mGy}^{*} \mathrm{~cm}$, representing $85.7 \%$ of the maximum dose encountered in the MDCT group. 
Table 2: Qualitative image scoring results for fine anatomic structures and metallic artifacts

\begin{tabular}{|c|c|c|c|}
\hline Scale Points & СВCT Group $(n=32)$ & MDCT Group $(n=19)$ & $P$ Value \\
\hline \multicolumn{4}{|l|}{ Cochlear inner wall } \\
\hline Not visualized (0) & $1(3 \%)$ & $0(0 \%)$ & $>.99$ \\
\hline Barely visualized (1) & $3(9 \%)$ & $2(10.5 \%)$ & \\
\hline Well-visualized (2) & $6(19 \%)$ & $3(15.8 \%)$ & \\
\hline Perfectly visualized (3) & $22(69 \%)$ & $14(73.7 \%)$ & \\
\hline \multicolumn{4}{|l|}{ Cochlear lateral wall } \\
\hline Not visualized (0) & $0(0 \%)$ & $1(5 \%)$ & .001 \\
\hline Barely visualized (1) & $0(0 \%)$ & $0(0 \%)$ & \\
\hline Well-visualized-(2) & $2(6 \%)$ & $8(42 \%)$ & \\
\hline Perfectly visualized (3) & $30(94 \%)$ & $10(53 \%)$ & \\
\hline \multicolumn{4}{|l|}{ Modiolus } \\
\hline Not visualized (0) & $0(0 \%)$ & $0(0 \%)$ & .37 \\
\hline Barely visualized (1) & $2(6 \%)$ & $0(0 \%)$ & \\
\hline Well-visualized (2) & $5(16 \%)$ & $1(5 \%)$ & \\
\hline Perfectly visualized (3) & $25(78 \%)$ & $18(95 \%)$ & \\
\hline \multicolumn{4}{|l|}{ Osseous spiral, lamina } \\
\hline Not visualized (0) & $18(56 \%)$ & 19 (100\%) & .002 \\
\hline Barely visualized (1) & $10(31 \%)$ & $0(0 \%)$ & \\
\hline Well -visualized (2) & $4(13 \%)$ & $0(0 \%)$ & \\
\hline Perfectly visualized (3) & $0(0 \%)$ & $0(0 \%)$ & \\
\hline \multicolumn{4}{|l|}{ Mastoid facial, canal wall } \\
\hline Not visualized (0) & $4(13 \%)$ & $4(21 \%)$ & .07 \\
\hline Barely visualized (1) & $10(31 \%)$ & $6(32 \%)$ & \\
\hline Well-visualized (2) & $9(28 \%)$ & $9(47 \%)$ & \\
\hline Perfectly visualized (3) & $9(28 \%)$ & $0(0 \%)$ & \\
\hline \multicolumn{4}{|l|}{ Metallic artifacts } \\
\hline Very strong artifact (0) & $0(0 \%)$ & $0(0 \%)$ & $<.001$ \\
\hline Strong artifacts (1) & $6(18.8 \%)$ & $14(74 \%)$ & \\
\hline Moderate artifacts (2) & $22(68.8 \%)$ & $5(26 \%)$ & \\
\hline Weak artifacts (3) & $4(12.5 \%)$ & $0(0 \%)$ & \\
\hline
\end{tabular}

Table 3: Qualitative image scoring results for electrode evaluation

\begin{tabular}{lccc}
\hline \multicolumn{1}{c}{ Scale Points } & CBCT Group $(\boldsymbol{n}=32)$ & MDCT Group $(\boldsymbol{n}=19)$ & $\boldsymbol{P}$ Value \\
\hline Electrode scalar position & & & \\
Not visualized (0) & $0(0 \%)$ & $0(0 \%)$ & .046 \\
Barely visualized (1) & $4(13 \%)$ & $3(16 \%)$ & \\
Well-visualized (2) & $3(9 \%)$ & $7(37 \%)$ & \\
Perfectly visualized (3) & $25(78 \%)$ & $9(47 \%)$ & \\
Single electrode contact & & & \\
$\quad$ visibility & $1(3 \%)$ & $10(52.6 \%)$ & $<.001$ \\
Not visualized (0) & $13(41 \%)$ & $6(31.6 \%)$ & \\
Barely visualized (1) & $11(34 \%)$ & $0(0 \%)$ & \\
Well-visualized (2) & $7(22 \%)$ & $3(15.8 \%)$ & \\
Perfectly visualized (3) & & & \\
Insertion angle of electrode & $0(0 \%)$ & $0(0 \%)$ & \\
Not measurable (0) & $32(100 \%)$ & $19(100 \%)$ & \\
Measurable (1) & Mean, $437.7^{\circ}\left[\mathrm{SD}, 120.8^{\circ}\right]$ & Mean, $329.4^{\circ}\left[\mathrm{SD}, 80^{\circ}\right]$ & \\
\end{tabular}

\section{DISCUSSION}

The number of CI operations has increased notably in recent years; however, there is still a wide spectrum of postoperative outcomes. Hence, more improvement in the electrode design, surgical approaches, and evaluation of the related detailed cochlear anatomy is necessary. ${ }^{5,20}$ Conventional radiography and MDCT have long been used for postoperative evaluation. CBCT imaging is a relatively new technique in the imaging of CIs and is reported to have a better spatial resolution than MDCT, with fewer metallic artifacts affecting the implant evaluation. ${ }^{3,21}$
High-resolution images are necessary for the proper visualization of single electrode contacts. Verbist et $\mathrm{al}^{22}$ compared 4 different MDCT devices in the evaluation of CIs. They used an in-plane resolution ( $x$ and $y$ axis) of $0.48-0.68 \mathrm{~mm}$ and a longitudinal resolution (z-axis) of $0.7-0.98 \mathrm{~mm}$, obtaining images with the high resolution necessary to adequately discriminate among the different electrode contacts. However, the use of flat panel detectors in CBCT devices provides images with higher isotropic resolution using a submillimeter detector size ranging from 0.09 to $0.4 \mathrm{~mm} .{ }^{15}$ In the current study, the voxel size used in the CBCT group was $0.125 \mathrm{~mm}$ (in all dimensions), which provided an isotopic image with high spatial resolution, thus allowing good-to-perfect visualization of single electrode contacts in 56\% of patients (including widely spaced and narrowly spaced electrode contacts) compared with only $16 \%$ in the MDCT group (including only widely spaced contacts).

The metallic artifacts were also significantly lower in the CBCT group than in the MDCT group $(P<.001)$. However, some of the patients undergoing CBCT implanted with CI532 (intercontact distance of $0.6 \mathrm{~mm}$ ) and CI512 (nonuniform intercontact distance ranging from 0.4 to 0.8 ) had high to-moderate metallic artifacts, leading to poor visualization of electrode contacts. The signal-to-noise ratio was significantly lower in the CBCT group; this finding can be explained by the higher scattered radiation of CBCT, which is one of its main disadvantages, which decreases the contrast resolution and increases image noise. ${ }^{8}$ The other disadvantage of CBCT was the relatively longer exposure time, which may increase the risk of motion artifacts. However, in patients with dizziness, shortened techniques with slightly lower resolution can be used to overcome this.

Some studies have examined the role of CBCT in the assessment of postoperative CIs in vitro. ${ }^{12,13}$ These studies compared the results of image analysis, including scalar positioning of the electrode, the presence of kinking, the number of intracochlear electrode contacts, and proper overall insertion using CBCT with those of histopathologic examinations in temporal bone specimens and concluded that CBCT as a noninvasive approach yielded results comparable with 


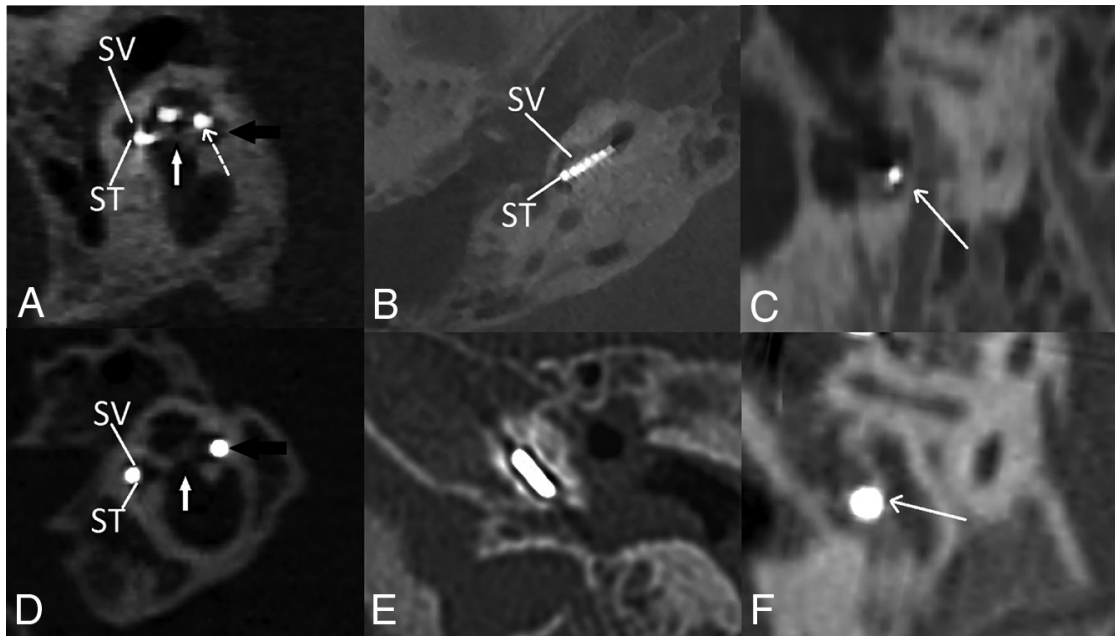

FIG 2. CBCT group. A, Midmodiolar view (cochlea) shows the modiolus (white arrow), the perfectly visualized cochlear lateral wall (black arrow), and the scalar translocation from ST to SV at the pars ascendens (dashed arrow). B, Axial view (cochlea) shows good visualization of single electrode contacts, $C$, Sagittal oblique view shows good visualization of the facial nerve canal wall (arrow). D, In the MDCT group, the midmodiolar view (cochlea) shows the modiolus (white arrow), nonvisualized cochlear lateral wall (artifacts) (black arrow), and the difficult scalar localization of the electrode (mostly ST). E, Axial view (cochlea) shows difficult identification of single electrode contacts and the osseous spiral lamina. F, Sagittal oblique view shows difficult identification of the facial nerve canal wall (arrow). ST indicates scala tympani; SV, scala vestibuli.

Table 4: SNR comparison

\begin{tabular}{lccc}
\hline & CBCT Group & MDCT Group & $\boldsymbol{P}$ Value $^{\mathrm{a}}$ \\
\hline SNR in bone (median) (IQR) & $8.31(3.8)$ & $10.77(4.25)$ & .02 \\
SNR in air (median) (IQR) & $6.58(3.85)$ & $11.97(5.53)$ & .001 \\
\hline
\end{tabular}

Note:-IQR indicates interquartile range.

${ }^{a}$ Mann-Whitney $U$ test.

those of histopathologic analyses, encouraging its clinical use. Razafindranaly et $\mathrm{al}^{14}$ compared the role of CBCT with that of MDCT in 9 patients in terms of scalar localization, insertion depth, and radiation doses. They concluded that there was good agreement between the 2 modalities in the evaluation of insertion depth; however, CBCT could be superior to MDCT in determining the scalar location of electrodes with lower doses.

Our results are in line with these previous studies because perfect visualization of the scalar position of the electrode was successful in $78 \%$ of cases in the CBCT group compared with $47 \%$ in the MDCT group. There was no statistically significant difference between both groups in the ability to measure the electrode insertion angle. Nevertheless, Jia et $\mathrm{al}^{23}$ had difficulty in assessing the scalar position of electrode or interscalar translocation using the mobile intraoperative CBCT device compared with previously obtained images using MDCT in the same patients; however, this issue could be attributed to the differences between mobile and fixed СBCT devices and the lack of specialized neuroradiologists during the operation.

The assessment of cochlear walls is essential for electrode localization, scalar position, and evaluation of postimplant inner ear trauma. Verbist et $\mathrm{al}^{22}$ concluded that the outer (lateral) cochlear wall could be better evaluated than the inner wall, a result attributed to the thick outer wall being a part of the otic capsule (dense bone), which provides a better contrast with the cochlear lumen, unlike the inner wall, which contains neural elements with lower density.

Our study revealed a statistically significant difference between the 2 groups regarding the visualization of the cochlear outer wall $(P<.001)$, with it being perfectly visualized in $94 \%$ of the patients of the CBCT group compared with 53\% in the MDCT group; however, there was no significant difference in the visualization of the cochlear inner wall $(P>.99)$ and the modiolus $(P=.37)$. Perfectly visualized cochlear inner walls and modioli were reported in $69 \%$ and $78 \%$ of patients in the CBCT group, respectively, compared with $73.7 \%$ and $95 \%$ in the MDCT group. This finding could be attributed to the larger number of lateral wall electrodes used in the MDCT group compared with the more perimodiolar and midscalar electrodes used in the CBCT group in our study population. The proximity of the electrode to the lateral cochlear wall or the modiolus can degrade the image quality by metallic artifacts. Furthermore, the osseous spiral lamina could be identified in only the CBCT group, in which $31 \%$ were barely visualized and $13 \%$ were well-visualized. The visualization of the spiral lamina could be further used for better evaluation of electrode translocation and inner ear trauma after CI.

The facial nerve canal, though an extracochlear anatomic structure, is an important surgical landmark in the CI field. ${ }^{24}$ In the current study, the good-to-perfect visualization of the facial canal bony wall was higher in the $\mathrm{CBCT}$ group than in the MDCT group. Although the difference was nonsignificant, this finding might allow a better radiologic evaluation of postoperative complications such as facial nerve injury or stimulation.

Many studies have compared the radiation dose of MDCT and CBCT in the assessment of CIs. ${ }^{25}$ Guberina et $\mathrm{al}^{26}$ compared CBCT with MDCT with 3 different machines (128-, 256-, and 384-multislice CT scanners) by imaging 4 temporal bone specimens and concluded that the dose-length product of a CBCT examination was $9 \%-15 \%$ of the dose-length product used during MDCT. In another study, this mean value was 200 [SD, 53.4] $\mathrm{mGy}^{*} \mathrm{~cm}$ for CBCT compared with 605 [SD, 57.2] $\mathrm{mGy}^{*} \mathrm{~cm}$ for MDCT. ${ }^{21}$ Our results also revealed significant radiation dose differences between the 2 groups. The dose-length product for the CBCT group ranged from 47.6 to $93 \mathrm{mGy}^{*} \mathrm{~cm}$, while it was $179-650 \mathrm{mGy}^{*} \mathrm{~cm}$ for the MDCT group, indicating a reduction of about $85.7 \%$ of the maximum dose with CBCT compared with MDCT. However, the low radiation dose is associated with a decreased signal-to-noise ratio, which makes $\mathrm{CBCT}$ 
unsuitable for soft-tissue imaging and limits its use for visualizing bone details. ${ }^{7}$

Other studies examined the possibility of using low-dose CT in the cadaveric lamb model for assessment of the electrode position. ${ }^{27,28}$ They succeeded in evaluating the electrode position using a reduced tube current of 50\% without an increase in the artifacts. They stated that the use of low-dose CT protocols might have results comparable with those of CBCT, both in terms of image quality and radiation dose. However, further prospective studies are needed to substantiate this possibility.

The main limitations of our study were the heterogeneity in the age, the differences in the types of implanted electrodes, and the 2 imaging modalities not being compared in the same group of patients because of the risk of radiation exposure.

\section{CONCLUSIONS}

CBCT imaging in postoperative patients with CIs can provide images with high spatial resolution and fewer metallic artifacts compared with MDCT, with a relatively lower radiation dose.

Disclosures: Ibraheem M. Al-Dhamari—UNRELATED: Employment: Koblenz University.

\section{REFERENCES}

1. Vaid S, Vaid N. Imaging for cochlear implantation: structuring a clinically relevant report. Clin Radiol 2014;69:e307-22 CrossRef Medline

2. Fishman AJ. Imaging and anatomy for cochlear implants. Otolaryngol Clin North Am 2012;45:1-24 CrossRef Medline

3. Vogl TJ, Tawfik A, Emam A, et al. Pre-, intra- and post-operative imaging of cochlear implants. Rofo 2015;187:980-89 CrossRef Medline

4. Abd El Aziz TT, El Fiky L, Shalaby MH, et al. Radiological evaluation of inner ear trauma after cochlear implant surgery by cone beam CT (CBCT). Eur Arch Otorhinolaryngol 2019;276:2697-2703 CrossRef Medline

5. Diogo I, Walliczeck U, Taube J, et al. Possibility of differentiation of cochlear electrodes in radiological measurements of the intracochlear and chorda-facial angle position. Acta Otorhinolaryngol Ital 2016;36:310-16 CrossRef Medline

6. Dhanasingh A, Jolly C. An overview of cochlear implant electrode array designs. Hear Res 2017;356:93-103 CrossRef Medline

7. Miracle AC, Mukherji SK. Cone beam CT of the head and neck, Part 2: clinical applications. AJNR Am J Neuroradiol 2009;30:128592 CrossRef Medline

8. Miracle AC, Mukherji SK. Conebeam CT of the head and neck, Part 1: physical principles. AJNR Am J Neuroradiol 2009;30:1088-95 CrossRef Medline

9. Wanna GB, Noble JH, McRackan TR, et al. Assessment of electrode placement and audiological outcomes in bilateral cochlear implantation. Otol Neurotol 2011;32:428-32 CrossRef Medline

10. Güldner C, Weiss R, Eivazi B, et al. Intracochlear electrodeposition: evaluation after deep insertion using cone-beam computed tomography [in German]. HNO 2012;60:817-22 CrossRef Medline

11. Cushing SL, Daly MJ, Treaba CG, et al. High-resolution cone-beam computed tomography: a potential tool to improve atraumatic electrode design and position. Acta Otolaryngol 2012;132:361-68 CrossRef Medline

12. Marx M, Risi F, Escudé B, et al. Reliability of cone-beam computed tomography in scalar localization of the electrode array: a radio histological study. Eur Arch Otorhinolaryngol 2014;271:673-79 CrossRef Medline
13. Saeed S, Selvadurai $\mathrm{D}$, Beale $\mathrm{T}$, et al. The use of cone-beam computed tomography to determine cochlear implant electrode position in human temporal bones. Otol Neurotol 2014;35:1338-44 CrossRef Medline

14. Razafindranaly V, Truy E, Pialat JB, et al. Cone Beam CT versus multislice CT: radiologic diagnostic agreement in the postoperative assessment of cochlear implantation. Otol Neurotol 2016;37:1246-54 CrossRef Medline

15. Scarfe WC, Farman AG. What is cone-beam CT and how does it work? Dent Clin North Am 2008;52:707-30 CrossRef Medline

16. Diogo I, Franke N, Steinbach-Hundt S, et al. Differences of radiological artifacts in cochlear implantation in temporal bone and complete head. Cochlear Implants Int 2014;15:112-17 CrossRef Medline

17. Dragovic AS, Stringer AK, Campbell L, et al. Co-registration of cone beam CT and preoperative MRI for improved accuracy of electrode localization following cochlear implantation. Cochlear Implants Int 2018;19:147-52 CrossRef Medline

18. Pearl MS, Roy A, Limb CJ. High-resolution secondary reconstructions with the use of flat panel CT in the clinical assessment of patients with cochlear implants. AJNR Am J Neuroradiol 2014;35:1202-08 CrossRef Medline

19. Tada A, Sato S, Masaoka Y, et al. Imaging of the temporal bone in children using low-dose 320-row area detector computed tomography. J Med Imaging Radiat Oncol 2017;61:489-93 CrossRef Medline

20. Whiting BR, Holden TA, Brunsden BS, et al. Use of computed tomography scans for cochlear implants. J Digit Imaging 2008;21:32328 CrossRef Medline

21. Merklen F, Piron J, Sicard M, et al. Cone-beam computed tomography in children with cochlear implants: the effect of electrode array position on ECAP. Int J Pediatr Otorhinolaryngol 2017;92:27-31 CrossRef Medline

22. Verbist BM, Joemai RM, Teeuwisse WM, et al. Evaluation of $\mathbf{4}$ multisection CT systems in postoperative imaging of a cochlear implant: a human cadaver and phantom study. AJNR Am J Neuroradiol 2008;29:1382-88 CrossRef Medline

23. Jia H, Torres R, Nguyen Y, et al. Intraoperative cone-beam CT for assessment of intracochlear positioning of electrode arrays in adult recipients of cochlear implants. AJNR Am J Neuroradiol 2018;39:76874 CrossRef Medline

24. Young JY, Ryan ME, Young NM. Preoperative imaging of sensorineural hearing loss in pediatric candidates for cochlear implantation. Radiographics 2014;34:E133-49 CrossRef Medline

25. Rafferty MA, Siewerdsen JH, Chan Y, et al. Intraoperative conebeam CT for the guidance of temporal bone surgery. Otolaryngol Head Neck Surg 2006;134:801-08 CrossRef Medline

26. Guberina N, Dietrich U, Arweiler-Harbeck D, et al. Comparison of radiation doses imparted during 128, 256, 384 multislice CT-scanners and cone beam computed tomography for intra and perioperative cochlear implant assessment. Am J Otolaryngol 2017;38:64953 CrossRef Medline

27. Weisstanner C, Mantokoudis G, Huth M, et al. Radiation dose reduction in postoperative computed position control of cochlear implant electrodes in lambs: an experimental study. Int J Pediatr Otorhinolaryngol 2015;79:2348-54 CrossRef Medline

28. Theunisse HJ, Joemai RMS, Maal TJJ, et al. Cone-beam CT versus multi-slice CT systems for postoperative imaging of cochlear implantation: a phantom study on image quality and radiation exposure using human temporal bones. Otol Neurotol 2015;36:592-99 CrossRef Medline

29. Bennink E, Peters JP, Wendrich AW, et al. Automatic localization of cochlear implant electrode contacts in CT. Ear Hear 2017;38:e37684 CrossRef Medline 